\title{
Notas sobre arqueología funeraria judía en época medieval*
}

\author{
Jorge Casanovas Miró
}

La investigación de los cementerios hebráicos ha constituido hasta hace muy poco tiempo un sector marginal e incluso marginado que despertaba escaso interés tanto por parte de las instituciones como de los investigadores. Las razones habia que buscarlas probablemente en las dificultades derivadas de la búsqueda y selección de un lugar donde excavar y en la escasez de materiales obtenidos sobre la que sentar las bases de un estudio mínimamente riguroso. Por otra parte ni en la $\mathrm{Pe}$ nínsula Ibérica, ni en Francia, Inglaterra o Italia se conservan cementerios judios de época medieval. En el caso de Inglaterra la cuestión se agrava al no conservarse ni siquiera restos epigráficos ${ }^{1}$.

Antes de entrar propiamente en materia conviene, sin embargo, hacer un balance del estado actual de nuestros conocimientos sobre los cementerios judíos.

El cementerio judío responde a la necesidad que tiene la comunidad de disponer de un lugar inviolable donde el cuerpo pueda descansar en paz en espera de la resurrección. Grande era la importancia del cementerio para el judío cuando en muchos casos éste era adquirido incluso antes que la propia sinagoga.

La necrópolis se encuentra en algunos casos a una distancia considerable de ciudades y pueblos, en la ladera de un monte ${ }^{2}$ bajo la protección aparente de un castillo ${ }^{3}$ o también extramuros, en una zona in-

* Comunicación presentada en el Encuentro Internacional de Historiadores «En torno a Sefarad". Toledo 1991.

AdLef, M., Jews of Medieval England. London 1939, pág. 182. Al efectuar en 1860 excavaciones en una escuela de Bristol apareció cierto número de lápidas con inscripción hebrea de las que actualmente nada se sabe pues no parece que nunca fueran publicadas. Recoge algunas noticias sobre cementerios judios en Inglaterra, Roth, C., A History of the Jews in England. Oxford 1979, pág. 13, n. ${ }^{\circ} 2$.

2 Así lo confirma la toponimia: Montjuic, Tumulus iudeorum, Judenberg o Jewbury.

3 Del mismo modo que algunos de los barrios judios buscan este sentimiento de protección alzándose al pie del castillo, muchas necrópolis parecen compartir idéntico sentimiento. 
mediata a la ciudad, donde el crecimiento de la misma tenderá a afectario con mayor facilidad que en el caso anterior, cubriendo sus restos con las nuevas construcciones.

El número de los cementerios era inferior al de las localidades donde la presencia de judíos aparece documentada. Las fuentes hacen referencia a judios que vivian en pequeños núcleos que no habian desarrollado todas las instituciones propias de una aljama perfectamente constituida y que llevaban a enterrar sus difuntos a la localidad más próxima donde existiera uno, empresa no siempre fácil ${ }^{4}$.

A diferencia del cementerio musulmán, sabiamente integrado en el pajsaje que lo rodea ${ }^{5}$, el cementerio judio se muestra como un espacio cerrado por un muro, inútil barrera contra las no poco frecuentes agresiones, del que sólo se conocen algunos datos a través de las fuentes documentales $^{6}$.

Muy pocos son los detalles que se conocen de su estructura interior. Por la disposición de los enterramientos en el cementerio de Barcelona, parece deducirse la existencia de un sector más antiguo a partir del cual se iría ocupando el espacio disponible con nuevas tumbas, cada vez más próximas unas de otras, en un intento de aprovechar al máximo un espacio que a todas luces resultaba cada vez más insuficiente. En el caso de Toledo, la transcripción de algunos epitafios, junto con las breves notas

4 Véase p.e. el caso de Mosse de Mana, de Forcalquier, que, cum nullum Forchalquerio haberent Forchalquerienses judei cimiterrium, llevaba a un niño en su ataúd para enterrarlo en Manosque. Al detenerse para descansar y beber un poco de agua en un pozo fue acusado de querer arrojar el cuerpo del niño al agua y de este modo emponzoñarla. (1306). SHATZMILLER. J., Recherches sur la communauté juive de Manosque au Moyen Age (1241-1329). París-La Haye 1973, págs. 133-135. Con mayor frecuencia se hallan referencias documentales sobre concesiones reales o autorizaciones a los judios para que puedan disponer en el futuro de un lugar para enterrar a sus muertos. Es entre muchos otros el caso de Burriana donde los judios se veian obligados a efectuar el penoso traslado de sus difuntos hasta Murviedro (1326). Doñate, J., y Magadalena. J.R., Three Jewish Communities in Medieval Valencia, Castellón de la Plana, Burriana, Villarreal. Jerusalem 1990, págs. 198-199.

s Toraes Balbás, L., "Cementerios hispanomusulmanes", Al-Andalus, XXII, 1957, págs. 132136

- Así se especifica en el documento de compra de un terreno para ser usado como cementerio en Vic (1327), Corbella, R., La aljama de juheus de Vich. Vic 1909, págs. 25-29 y doc. 3 y 4 ; en Tortosa el muro era de piedra en la parte inferior y de tapial en la superior (hasta 1396), Curto. A., "El cementiri jueu de Tortosa” en Actes del Primer Col.loqui d'Història dels Jueus a la Corona d'Aragó. Lleida 1991, págs. 401-409; en Calatayud (1483-84) un muro separaba el cementerio de los judios del de los conversos, MaRín. E., "Relación judeoconversa durante la segunda mitad del siglo xV en Aragón: enfermedades y muertes", Sefarad, XLIII, 1983, pág. 327. En Francia se documenta su existencia en Sens, Mâcon (1309), Dijon (1310) y en Brie-Comte-Robert (Seine-et-Marne) (1331), NaHON, G., "Les cimetières" en Art et archéologie des Juifs en France médiévale. Toulouse 1980, pág. 78. 
que la acompañan, realizada en el siglo $x \mathrm{~V} \mathrm{I}^{7}$, tampoco permite conocer si en este cementerio existian concentraciones de miembros de una misma familia en un mismo sector. El copista transcribió los textos al azar sin seguir un determinado orden.

Las localidades más importantes podian disponer de más de un cementerio en función de las necesidades concretas generadas por las circunstancias del momento o por el volumen de población judía que acogía $^{8}$.

En relación al uso funerario propiamente dicho, el cuerpo se halla en posición de decúbito supino, con los brazos extendidos a lo largo del cuerpo o doblados sobre distintos puntos. Sólo excepcionalmente aparecen cruzados sobre el pecho. La cabeza puede estar inclinada bien sobre el hombro derecho o el izquierdo. Los cadáveres están orientados con los pies hacia Levante ${ }^{9}$, aunque se constatan variantes incluso dentro de una misma necrópolis ${ }^{10}$. Se da el caso incluso de presentar la cabeza orientada hacia el Norte ${ }^{11}$.

Por regla general no presentan ajuares o en caso contrario éstos son muy escasos limitándose a algunos anillos de oro, plata o bronce, con o sin inscripción, decorados con elementos vegetales, figurativos o simplemente motivos en zigzag que ofrecen curiosos paralelos y semejanzas entre sí. Otros objetos de adorno aparecen con relativa frecuencia en las necrópolis hispanas: colgantes, pendientes, zarcillos, cuentas de collar y otros adornos, cerámica o clavos ${ }^{12}$. 335-337.

7 LEÓN, P., "Judíos toledanos víctimas de la Peste Negra”, Sefarad, XXXVII, 1977, págs.

${ }^{8}$ Por lo que se refiere a Barcelona no es posible deducir a partir de la documentación si existían dos cementerios o un sector viejo y un sector nuevo de un mismo cementerio. En Lérida hubo con toda seguridad dos necrópolis, aun cuando la documentación permite suponer incluso la existencia de un tercero, BERTRÁN, P., "Documento sobre un nuevo cementerio judio en Lérida (1383)", Sefarad, XLI, 1981, págs: 114-120. En Toledo los escasos restos epigráficos hallados «in situ» y la documentación parecen confirmar que hubo dos necrópolis, una en el "Cerro de la Horca", donde fueron reaprovechados materiales de un cementerio musulmán del siglo $\times 1$, y otra en la zona de la Fábrica de Armas, en el sector de la Vega más cercano al rio. En París son mencionados tres cementerios entre 1258 y 1327 , “Inventaire archéologique" en Art et archéologie des Juits en France médievale. Toulouse 1980, pág. 363.

9 Casanovas, J., "Necrópolis judias medievales de la Península lbérica», Revista de Arqueologia, n. ${ }^{\circ} 71,1987$, págs. 53-54.

10 De las 10 sepulturas excavadas en 1953 en Teruel, todas seguian una orientación NOSE, salvo la $H$, algo alejada del resto, perfectamente orientada O-E, NOVELLA, A., "Informe sobre la necrópolis judaica de Teruel y sus recientes exploraciones". Teruel, n. ${ }^{\circ} 10,1953$, págs. $257-$ 261.

TARACENA, B., "Cadáveres atravesados por clavos en el cementerio judio de Deza (Soria)", Investigación y Progreso, $7,1933, n .^{\circ} 3$, págs. 65-71.

12 Casanovas, J., cit. supra, pág. 54. En colaboración con RIPOLL, O., “Catálogo de los 
Por el exterior se señalizaba la sepultura mediante una lápida con inscripción que normalmente se colocaba al cumplirse un año del sepelio, si bien en algunos casos ésto no era posible como sucedió en Carpentras, donde las autoridades cristianas prohibieron durante un tiempo poner inscripción en la piedra funeraria ${ }^{13}$. En última instancia no debe descartarse la existencia de epitafios sobre materiales perecederos como por ejemplo la madera ${ }^{14}$.

Salvo en el caso del Montjuic de Barcelona, no se ha realizado un estudio antropológico in extenso de los restos aparecidos en las excavaciones ${ }^{15}$.

Para el estudio de las necrópolis hebreas es preciso contar con tres tipos de fuentes: documentales, epigráficas y arqueológicas.

La documentación aporta en la mayoría de los casos una información de carácter muy limitado e impreciso, centrada ante todo en aspectos legales:

- Permisos y prohibiciones para crear nuevos cementerios.

- Litigios por su posesión (las primeras noticias de dos de los tres cementerios hebreos del París medieval, 1283 (1) y 1258 (2), tratan precisamente de esta cuestión).

- Recuperación de antiguas necrópolis tras períodos de desastre o debido a confiscaciones arbitrarias.

- Pago de los censos anuales estipulados.

materiales aparecidos en la necrópolis judaica de Deza (Soria)”, Celtiberia, 65, 1983, págs. 135 148.

Nahon, G., cit. supra, pág. 75 . Dicha prohibición fue renovada el 25 de septiembre de 1751 por Benedicto XIV y por el obispo d'Inguimbert.

4 Raphael, F., y Weyl, R., Juifs en Alsace. Culture, société, historie. Toulouse 1977, págs. 158-160. La estela de madera de Hegenheim de 1855 permite suponer la existencia de una antigua tradición sobre el uso de dichas estelas que podría explicar la ausencia en Alsacia de estelas de los siglos $x \vee$ y $x v i$. Por extensión nada nos impide creer en una tradición similar en cualquier otro punto de Europa occidental.

15 Prevosti. A., "Estudio tipológico de los restos humanos hallados en la necrópolis judaica de Montjuich (Barcelona), Sefarad, XI, 1951, págs. 75-90. M. a y Prevosti. A., "Restos humanos procedentes de una necrópolis judaica de Montjuich (Barcelona)", Trabajos del Instituto "Bernardino de Sahagún" de Antropologia y Etnologia, XII, 1951, págs. 65-148. Hay que aceptar con reservas la clasificación como hebrea de la necrópolis localizada en 1988 en Santa Coloma de Queralt (Tarragona), GenerA, M., LalueZA, C. y GimÉnez. S., “Aportacions a la historia de Santa Coloma de Queralt: Dades sobre una necrópolis excavada en el nucli urbá", Recull Mateu Fletxa "El Vell» (1481-1553), Estació de Recerca Bibliográfica i Documental "Margalló del Balcó." Tarragona 1992, págs. 35-48. El mal estado de los restos humanos aparecidos en la breve exploración llevada a cabo en la necrópolis judia de Biel y lo reducido de la intervención sólo permiten fijar a grandes rasgos las características de los individuos, LANZAROTE, M. ${ }^{\text {a }} \mathrm{P}$., "La necrópolis judia de Biel (Altas Cinco Vitlas, Zaragoza)", Suessetania, n. ${ }^{\circ} 12,1992$ págs. $72-73$. 
- Medidas de protección de los mismos.

- En un último apartado pueden incluirse todas aquellas referencias indirectas derivadas de la venta de terrenos colindantes con el cementerio. En muchas ocasiones la información facilitada es lo suficientemente amplia como para que sea posible ubicar la necrópolis dentro de un sector determinado.

Desde la segunda mitad del siglo XIV y hasta mediados del siglo XV, especialmente en la Península Ibérica; se advierte un incrementeo de la aportación documental en la que se refleja de un modo muy claro el aumento de la conflictividad entre las distintas comunidades judias y las instituciones y autoridades locales por la posesión de los cementerios.

Consumada la expulsión, o las expulsiones, los pleitos por la posesión de los cementerios y las piedras de los mismos no cesaron, dando pie a nueva documentación sobre su venta y posterior liquidación ${ }^{16}$.

Acerca de lo anteriormente expresado y sobre las vicisitudes que tuvieron que sufrir los judíos y sus propiedades es esclarecedor el caso de la necrópolis hebrea de Jerez de la Frontera. Los pormenores de esta historia, común por otra parte a muchos otros lugares, permiten demostrar las dificultades que pueden derivarse de la investigación arqueológica en un lugar tan sumamente alterado ya desde antiguo.

Los judíos adquirieron la propiedad de un terreno para ampliar su cementerio el 3 de marzo de 1383. Tras los alborotos de 1391 un grupo formado por 49 conversos donaron aquella propiedad al convento de Santo Domingo sin provocar con ello ninguna reacción por parte de los judios. Ya en posesión de ella los Dominicos y cuando ya habian iniciado el proceso de su parcelación para obtener de este modo un rendimiento económico, el Concejo de Jerez la embargó, pues la zona formaba parte del sector en expansión de la ciudad. Cuando intervino la justicia fue anulado el embargo y el monasterio pudo proseguir las obras en pleno uso de sus derechos. Los judios por su parte debían conformarse con el sector antiguo de su cementerio. A mediados del siglo xV la expansión de la ciudad, al encontrarse el cementerio inmediato a la muralla y la creciente animosidad hacia los judios rompió la aparente tranquilidad que existía entre ambas comunidades. El 2 de julio de 1459 los alcaldes mayores de Jerez y algunos de los regidores y jurados concedian a Bartolomé Fernández Catalán un solar situado en medio de los dos caminos

16 Estamos preparando en colaboración con M. ${ }^{a}$ Cinta Mañé la publicación de los documentos sobre la venta del cementerio judio de Barcelona a finales del siglo xiv. 
que llevaban respectivamente al monasterio de Santo Domingo y al cementerio judio, lo que provocó las protestas de la comunidad judía que se amparaba en un cédula regia que entre otras cosas exigía la salvaguarda de sus enterramientos. Tras largas deliberaciones el Concejo anuló la anterior donación dando con ello la razón a los judíos. El 26 de marzo de 1460, siendo alcalde Juan de Buñuelo, éste solicitó del concejo que, ya que le habían elegido para este cargo, le hicieran merced de un solar en el cementerio de los judíos para edificar en él. Esta nueva acción motivó la intervención de las autoridades de la aljama con una propuesta singular. Ellos cedían generosamente el solar motivo del pleito a condición de que no volviera a privárseles de una nueva parte de su cementerio. Esto fue en vano, pues el 20 de abril de 1460 el concejo decidió conceder parcelas del cementerio a todos aquellos que quisieran tomarlas a tributo para edificar en ellas sin tener en cuenta la condición exigida por los judíos. En este momento Bartolomé Fernández Catalán, al ver el sesgo que tomaban los acontecimientos, protestó a su vez exigiendo el solar que anteriormente le había sido concedido, obteniéndolo esta vez. Nuevas protestas de los judios no dieron ningún fruto. Noticias dispersas fechadas en 1483 permiten conocer que la aljama se había quedado con una parte muy mermada de su cementerio y que por otra parte tampoco se había llevado a término la pretendida ampliación urbanística del sector, pues muchos de los que habían tomado aquellas parcelas a tributo no habian edificado aún en ellas ${ }^{17}$.

Desafectados por éstas y por otras causas, los cementerios se convierten en canteras improvisadas de las que se extraen los bloques con o sin inscripción para ser reutilizados. Es muy poco frecuente que las piedras permanezcan en el mismo lugar o en sus inmediaciones, aunque puede ocurrir algunas veces. Este es el caso de las lápidas halladas al excavar en 1847 los cimientos de la editorial Hachette en París ${ }^{18}$. En Barcelona de las 60 inscripciones conservadas, sólo dos se encontraban sobre la sepultura, cinco en el sector del cementerio y el resto reutilizado en diversas construcciones de la ciudad ${ }^{19}$.

Las piezas de menor tamaño son transportadas incluso a distancias considerables $^{20}$ y convenientemente fragmentadas son reutilizadas con

17 Sopanis, H. S. de, "Contribución a la historia de la juderia de Jerez de la Frontera", Sefarad, XI, 1951, pág. 349-370.

18 NaHON. G., Inscriptions hébraiques et juives de France médiévale." París 1986, págs. 47. 148. Para más detalles sobre este hallazgo cf. "Rapport sur les inscriptions hebraiqques de la France", Nouvelles Archives des Missions Scientifiques et Littéraires, XII, 1904 págs. 238-241.

19 Casanovas. J., El cementerio judio de Montjuic (Barcelona), Catálogo de sus inscripciones (en prensa).

${ }_{20}$ El cementerio judío de Gerona constituye un ejemplo notable por la dispersión de sus 
diversos fines: para cerrar un pozo ${ }^{21}$, fuentes ${ }^{22}$, para marcar los límites de una propiedad ${ }^{23}$, material de construcción en murallas ${ }^{24}$, casas o palacios ${ }^{25}$. Cuando ésto sucede uno de los problemas que se plantean es el de adscribir dichas piezas a una necrópolis determinada, ya que puede ocurrir que han sido reutilizadas en un lugar donde no hubo cementerio judio y a cierta distancia del más próximo.

Los restos epigráficos conservados constituyen tan sólo una mínima parte del total existente en su momento. El corpus de inscripciones hebreas de algunos países arroja unas cifras realmente bajas y si nos atenemos a ciertos casos muy concretos el balance es extraordinariamente decepcionante ${ }^{26}$. A partir de los trabajos de G. Nahon se puede observar la desproporción evidente entre el número de cementerios que debieron existir y los restos que de los mismos se conservan. En Provenza hay 43 localidades en las que está probada documentalmente la presencia de judíos. Los textos sólo aluden a la existencia de 14 cementerios y de todos ellos sólo se conserva un único resto epigráfico. Algo parecido ocurre en el Franco Condado donde de las 80 localidades con judíos no se conserva ningún resto de los cinco cementerios documentados.

A partir de estos datos resulta evidente la imposibilidad de realizar estadísticas fiables dado el escaso número de elementos disponibles y

materiales, CASAnOvas, J., "Lapides de Montjuic, amb inscripcions hebraiques, reutilitzades a la rodalia de Girona", Cal/s, 3, 1988-1989, págs. 35-44.

${ }_{21}$ WEYL. R., "Les inscriptions hebraïques des Musées de Strasbourg», Cahiers Alsaciens d'Archéologie, d'Art et d'Histoire, XVIII, 1974, págs. $130-131, n .{ }^{\circ} 5$.

22 NAHON, G., Inscriptions..., pág. 327-328, n. 281 (Ennezat). En una propiedad situada en el mismo término otra lápida había sido reutilizada como fregadero, pág. $329, \mathrm{n}^{\circ} 283$.

${ }^{23}$ Uno de los grandes bloques con inscripción del cementerio hebreo de Gerona que quedaron abandonados entre la vegetación de los márgenes del torrente llamado Bou d'Or fue reutilizado como hito de una propiedad del Mas Coll de Pont Major

${ }^{24}$ En 1349, tras la destrucción de las comunidades judias renanas, las piedras de los cementerios judios de Colonia fueron reutilizadas para reconstruir los castillos de Lechenich y el de Hulchrath. En la segunda mitad del siglo xv se construyó una fortificación frente a la Puerta Severin utilizando las lápidas del cementerio inmediato como material de construcción. KoBER. A., "Jewish Monuments of the Middle Ages in Germany", Proceedings of the American Academy for Jewish Research, XV, 1945, pags. 19-24 ("The Fate of the Tombstones from the Jewish Cemetery at the Bonn Gate»)

25 En los muros del antiguo palacio del Lugarteniente, sede del Archivo de la Corona de Aragón, en Barcelona han sido localizados 14 fragmentos de inscripciones hebreas procedentes del cementerio de Montjuic. Casanovas, J., El cementerio judío....

26 KoBER. A., cit supra, recoge 110 inscripciones de Nuremberg. Worms, Colonia y Speyer con fechas que van desde el año 1085 al 1428; Sólo 29 de época medieval en Hungria, cf. SCHEIBER, A., Jewish Inscriptions in Hungary. Leiden 1983. Un total de 278 inscripciones en Francia de las que 110 corresponden a París, 45 a Dijon y 41 a Strasbourg, cf. NaHON. G., Inscriptions hébraiqques... Por lo que se refiere a España incluimos un total de 255 piezas en el catálogo de inscripciones funerarias que actualmente estamos elaborando 
no parece lógico esperar que en un futuro próximo dichas cifras puedan variar. En este campo más que en ningún otro el hallazgo responde al más puro azar y como ya señaló R. Etienne el azar no es selectivo ${ }^{27}$. Es por ello que, dada la precariedad de los materiales, cada fragmento por pequeño que sea adquiere un valor considerable aunque sólo sea desde el punto de vista testimonial.

Otro de los problemas y no el menos importante es el de la datación, especialmente de los fragmentos. De las 110 inscripciones que constituyen la colección epigráfica habraica parisina, 74 no llevan explícita la fecha y las 36 restantes se reparten de una forma muy desigual, una del siglo XII, 34 del xill y una del XIV. Hay que advertir, sin embargo, que en ciertos casos, aun cuando dichas piezas puedan conservarse enteras, no se acostumbraba a indicar la fecha de la defunción. Es lo que ocurre con los conjuntos epigráticos de Dijón, Carpentras o Nancy, donde el texto de las inscripciones es muy breve. Del total de lápidas de la colección epigráfica barcelonesa, 70 incluyendo las actualmente desaparecidas, sólo un 20 por 100 lleva expresada la fecha de defunción. Dentro de este grupo hay cuatro de las que sólo sabemos que no son anteriores a 1240 , mientras que el resto abarca desde el siglo XI hasta el XIV sin rebasar el año 1307 (dos del siglo $\mathrm{xI}$, tres del $\mathrm{xII}$, tres del XIII y cuatro del xIV). Finalmente y aunque en menor grado conviene no olvidar la existencia de cómputos locales con ciertas oscilaciones en las fechas, aspecto éste aún no totalmente estudiado.

Para resolver éstas y otras cuestiones el epigrafista cuenta con ciertos elementos que en el estado actual de nuestros conocimientos no permiten un avance significativo de las conclusiones ni cuantitativas ni cualitativamente. Entre éstos figura la arqueología en primer lugar, aunque las intervenciones arqueológicas, muy escasas, no han servido para resolver los problemas que en estos casos concretos se planteaban. El estudio del soporte, su decoración, la paleografía del texto y el formulario poco parecen aportar de momento dada la pervivencia de tipos y fórmulas sin cambios significativos a lo largo de un dilatado período de tiempo. En última instancia poco es lo que aporta la onomástica, ya que no existe una coincidencia entre los nombres que figuran en la documentación y los de los epitafios. Los nombres se repiten constantemente y es poco frecuente la identificación de los personajes salvo cuando se trata de figuras relevantes.

27 ETIENNE, R., “Démographie et épigraphie», Atti del Terzo Congr. Int. di Epigrafia Greca e Latina. Roma 1957, págs. 415-424. 
La presencia de nombres sugestivos (Mons judaicus [Narbona], tumulus iudaeorum [Znaim], Jüdenbüchel [Colonia], etc.) unidos a un testimonio documental no siempre son garantía segura de la localización de una necrópolis hebrea. Varios son los ejemplos que pueden aportarse en este sentido. En las excavaciones efectuadas en la llamada Huerta del Rey con motivo de las obras de construcción del metro sevillano fueron localizadas 33 sepulturas de las que una era tardorromana, 31 musulmanas de los siglo $\mathrm{X}$ al $\mathrm{XI}$ y una podía ser judía aunque nada permitía confirmar$10^{28}$. En Córdoba 60 tumbas supuestamente hebreas con escasos ajuares formados por cerámica mudéjar y clavos tanto de los ataúdes como hincados en algunos miembros de los difuntos ${ }^{29}$. La "Cuesta de los Hoyos" de Segovia ha proporcionado un cierto número de cuevas y sepulcros antropomorfos sin posibilidad de identificación segura ${ }^{30}$. En Toledo existe una amplia zona de necrópolis en la que se incluyen tres cementerios musulmanes, un cementerio cristiano cerca del Cristo de la Vega, uno mudéjar y probablemente dos hebreos, uno en el "Cerro de la Horca", donde aparecieron dos inscripciones hebreas, y otro en la zona de la Fábrica de Armas. En este caso puede inducir a error el hecho de compartir tanto judíos como musulmanes tipos parecidos de enterramientos (lucillos) ${ }^{31}$.

Las excavaciones han afectado sólo pequeños sectores, nunca la totalidad de las necrópolis, lo que no ha permitido obtener datos representativos. En Barcelona se excavaron 171 tumbas entre 1945 y $1946^{32}$. Con posterioridad y de forma esporádica han aparecido nuevos restos sin cuantificar. En León, donde ya habian aparecido un cierto número de lápidas desde $1874^{33}$, fueron localizadas en 1954 varias hileras de tumbas (sic) $^{34}$ y cuatro más en 1973 en el jardín de una casa ${ }^{35}$. En Soria se

28 Fernandez. F. y Hoz, A. de la, “El cementerio judio de la Buhayra (Huerta del Rey, Sevilla)", Actas del I Congreso de Arqueología Medieval Española. Zaragoza 1986, vol. IV, págs. 49-72.

29 VázQuez, J. A., "La necrópolis hebraica de Córdoba”, Algo, VII, 1935, n. ${ }^{\circ}$ 299, págs. 15 16.

3o Burdiel, l., “Excavaciones en el antiguo fonsario u osario de los judíos (Segovia)", Noticiario Arqueológico Hispánico, VI (1962), 1964, págs. 216-226.

${ }^{31}$ Garcia, A de Juan, Los enterramientos musulmanes del circo romano de Toledo. Toledo 1987, pág. 11-19.

32 Durán i SAnpere, A. y Millás, J. M.", "Una necrópolis judaica en el Montjuich de Barcelona", Sefarad, VII, 1947, págs. 231-259.

33 Cantera, F. y Millás, J. M. ${ }^{a}$, Las inscripciones hebraicas de España. Madrid 1956, págs. 5-24

34 Cantera. F., “Nuevo hallazgo epigráfico en León», Sefarad, XIV, 1954, págs. 119-121.

35 Pérez Herrero, E. y Pérez Castro, F. «Puente Castro: Excavación de cuatro tumbas judias y hallazgo de un nuevo epitafio hebreo", Sefarad, XXXIV, 1974, págs. 31-41. 
encontró un número indeterminado de sepulturas en $1955^{36}$, así como 57 en Deza en $1930^{37}$. En Lérida venían produciéndose hallazgos desde el siglo XVIII en una zona en la que se mezclaban las sepulturas de épocas muy diversas ${ }^{38}$. Finalmente en Teruel aparecieron primero un número indeterminado de tumbas en $1926^{39}$. En 1953, 30 más y un número indeterminado, aún sin publicar, en la década de los sesenta al construir una guardería. La mayor parte de las excavaciones aquí reseñadas se llevaron a cabo aprovechando la realización de obras de diversa indole, especialmente de infraestructura. Los resultados así obtenidos reflejan sólo parcialmente una realidad que hasta que no sean efectuados trabajos de mayor envergadura apenas proporcionan una visión muy limitada de los mismos y por ello de difícil sistematización.

Fruto del interés que merece este aspecto de la investigación de la historia de los judíos en la Europa occidental y muy especialmente en España son los trabajos recientemente emprendidos por el Museo Sefardí de Toledo en la búsqueda de nuevos yacimientos o los proyectos de excavación de las necrópolis aragonesas de Biel y Uncastillo, aún pendientes de realización. Sólo el esfuerzo coordinado entre individuos e instituciones permitirá que en un futuro la arqueología judía ocupe el lugar que le corresponde dentro del campo de acción de los arqueólogos medievalistas.

${ }^{36}$ Ortego, T., «Piedras de historia. Un crismón medieval y una lauda hebrea en el castillo de Soria», Celtiberia, 10, 1955, pág. 311-314.

37 TARACENA, B., "Cadáveres atravesados por clavos en el cementerio judío de Deza", Investigación y Progreso, VII, 1933, págs. 67.

38 Romano, D., "Restos judios de Lérida», Sefarad, XX, 1960, págs. 50-65.

39 Floriano, A. C., "Hallazgo de la necrópolis judaica de la ciudad de Teruel", $B R A H$, LXXXVIII, 1926, págs. 845-851. 